\title{
Gradhiva
}

GRADHIV

Revue d'anthropologie et d'histoire des arts

11 | 2010

Grands hommes vus d'en bas

\section{Objets, personnes, esprits}

Alfred Gell, L'art et ses agents. Une théorie anthropologique, Bruxelles, Les presses du réel (« Fabula »), 2009

\section{Olivier Allard}

\section{(2) OpenEdition}

\section{Journals}

Édition électronique

URL : http://journals.openedition.org/gradhiva/1767

DOI : 10.4000/gradhiva. 1767

ISSN : 1760-849X

Éditeur

Musée du quai Branly Jacques Chirac

Édition imprimée

Date de publication : 19 mai 2010

Pagination : 207-213

ISBN : 978-2-35744-025-8

ISSN : 0764-8928

Référence électronique

Olivier Allard, «Objets, personnes, esprits », Gradhiva [En ligne], 11 | 2010, mis en ligne le 19 mai 2013, consulté le 19 avril 2019. URL : http://journals.openedition.org/gradhiva/1767 ; DOI : 10.4000/ gradhiva.1767

(c) musée du quai Branly 


\section{OBJETS, PERSONNES, ESPRITS}

Alfred Gell, L'art et ses agents. Une théorie anthropologique. Bruxelles, Les presses du réel ("Fabula»), 2009

L'Art et ses agents est un livre qui captive - comme un motif labyrinthique envoûte l'esprit d'un démon, comme un chef-d'œuvre fascine un spectateur - parce que l'argumentation, les rapprochements, les références, les analyses prennent toujours des tournures imprévues. Ce rapprochement est un peu convenu, et d'autres commentateurs ont déjà montré qu'il était possible de pasticher ainsi Alfred Gell en rendant compte de sa propre œuvre (Miller 2000 et, implicitement, Campbell 2001 : 117). Toutefois, la fascination n'est pas un processus automatique : alors que les débats dans le monde anglosaxon à propos des théories de Gell se sont largement apaisés, il a fallu attendre dix ans pour que l'ouvrage soit traduit en français, publié par Les presses du réel dans la collection «Fabula » que dirige Alexandre Laumonier. Les traducteurs, Sophie et Olivier Renaut, se sont attaqués à une tâche complexe, car le style élégant et souvent ironique de Gell s'assortit ici de nombreuses références techniques à des domaines très divers - anthropologie, philosophie, histoire de l'art, sciences cognitives, etc. II s'agit dans l'ensemble d'un succès admirable $^{1}$, et la qualité de la traduction autant que le soin apporté à la production du livre et à la présentation d'une riche iconographie offrent enfin au chef-d'œuvre de Gell une audience élargie en France, qu'il ne reste plus qu'à gagner.

Si l'ouvrage a été remarqué dans le monde universitaire, c'est notamment parce que Gell dit y proposer une théorie proprement anthropologique des artefacts artistiques, contre l'approche esthétisante classique. Mais il s'agit alors d'anthropologie sociale (suivant la tradition britannique], et certainement pas d'anthropologie culturelle (telle qu'on la pratique dans la tradition américaine). En effet, Gell veut situer les œuvres d'art dans des réseaux de relations sociales plutôt qu'interpréter leur signification et étudier les conceptions du beau développées dans différentes sociétés ${ }^{2}$. Une des thèses centrales de l'ouvrage, ou plutôt la manière dont on pourrait en résumer l'approche en une expression, consiste à dire qu'il faut traiter les objets comme des personnes (Gell 1998: 7, 2009 [1998] : 9). Si une telle affirmation peut désarçonner des historiens ou des théoriciens de l'art, elle s'enracine en revanche dans la longue tradition maussienne de l' $\varepsilon$ ssai sur le don (Mauss 1950 [1923-1924]). Toutefois, il faut remarquer

\section{-}

1. On ne peut donc que regretter quelques imprécisions de détail dans la traduction d'expressions techniques, comme person-enhancing (Gell 1998 : 199), rendu par «qui met en valeur l'humain » (Gell 2009 [1998] : 239) alors qu'il s'agit du concept anthropologique de «magnification de la personne »; ou mind-trap (Gell 1999 [1996]), une référence aux «pièges à pensée » de Pascal Boyer (1988) et, indirectement, de Pierre Smith [1979), qui est malencontreusement traduit par «casse-tête» [Gell 2009 [1998] : 100). Certains choix sont discutables, même s'il faut admettre qu'il n'existe pas de solution parfaite, par exemple «élargissement de l'esprit » pour extended mind (chap. IX] ou «personne disséminée» pour distributed person (chap. VII].

2. Certains débats techniques ont eu lieu à ce sujet; je veux les signaler sans pour autant les détailler: si Gell rejette l'approche sémiologique, quel statut donner à son usage des termes de Pierce (indice, prototype, etc.) ? Ne confond-il pas sans raison indice et icône? A-t-il raison de réduire la sémiologie à la langue, et la langue à un code ? etc. Voir notamment Layton 2003. que, en France, l'interprétation courante de Mauss a tempéré ce précepte : dans son «Introduction à l'œuvre de Marcel Mauss », Claude Lévi-Strauss mettait par exemple en garde contre le danger de prendre les conceptions indigènes à la lettre (1950: XLVI). En revanche, c'est précisément ce qu'ont choisi de faire, longtemps après, des anthropologues anglo-saxons comme Marilyn Strathern (1988), dont Gell se réclame explicitement. La caractéristique qu'habituellement seules les personnes possèdent, et que Gell étend aux choses, c'est l'agentivité (agency), une notion aux connotations multiples que l'on peut temporairement et sommairement définir comme une intentionnalité active ou agissante.

La plupart des critiques ont privilégié ces questions théoriques et les concepts déployés par Gell dans les premières pages de son ouvrage, donnant l'impression qu'il s'agit d'un traité purement théorique. De plus, sa conception de ce qu'est une théorie anthropologique pourrait faire croire à une dérive sociologisante, où les caractéristiques matérielles et formelles des artefacts disparaîtraient derrière l'analyse des réseaux sociaux ou des conceptions que les membres de diverses sociétés se font des objets. Certes, Gell dit vouloir traiter les objets comme des personnes, mais les anthropologues décrivent rarement en détail l'aspect physique des individus rencontrés sur le terrain... Or Gell accorde une place centrale à l'analyse précise d'artefacts artistiques (kolam, idoles, tatouages, statues, etc.), et il avait critiqué, dès ses travaux antérieurs, les approches sociologiques qui échouent à prendre en considération

\section{Alfred Gell}

Lart et ses agents, une théorie anthropologique

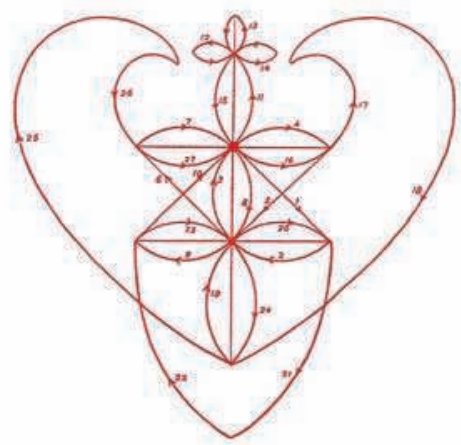

Les presses du réel 


\section{CHRONIQUE SCIENTIFIQUE NOTES CRITIOUES}

les œuvres d'art en elles-mêmes (1999 [1992] : 162]. À ne lire que certains passages, on pourrait le croire beaucoup plus proche des spécialistes d'esthétique qu'il critique que des anthropologues qui le discutent; mais les analyses qu'il conduit diffèrent alors, tant par leur nature que par leur intention, de celles d'autres anthropologues ou historiens spécialistes de l'art.

De nombreuses publications ont discuté et évalué l'approche de $\mathrm{Gell}^{3}$, et il est remarquable qu'il ait été pris entre deux feux : pour certains il serait allé trop loin et pour d'autres pas assez! Je vais discuter ces deux séries de critiques.

\section{Trop loin ou pas assez}

Les premières sont en général émises par des anthropologues qui travaillaient sur l'art avant la parution du livre de Gell. C'est notamment le point de vue d'Howard Morphy, qui parle d'une «analogie poussée trop loin » (2009: 6), et qui réduit la question à celle de savoir si les membres d'une société ou culture donnée «croient 》 que l'objet est capable d'actions intentionnelles, c'est-à-dire possède une agentivité. La radicalité de l'approche de Gell est alors singulièrement émoussée, la séparation entre la réalité et la manière dont on la conceptualise est restaurée. Au premier plan revient l'étude des conventions sociales ou des croyances culturelles de chaque société productrice d'artefacts artistiques. Une seconde question est celle de la spécificité des objets d'art. Gell ne définit pas son objet a priori, certains de ses exemples sont explicitement d'un autre ordre, et Morphy ou Layton rejettent cette dissolution de l'objet dont ils étaient - et sont encore - des spécialistes. Robert Layton (2003: 452) critique ainsi certains parallèles émis par Gell, qu'il juge «trompeurs » : lorsque Gell cite le cas des mines antipersonnelles posées par les soldats de Pol Pot comme un exemple d'agentivité étendue, Layton se révolte. Des mines ou des pièges n'exercent pas une agentivité de la même manière que des objets d'art - si les soldats avaient enterré des portraits de Pol Pot et non des mines, il y aurait moins d'infirmes au Cambodge -, et c'est précisément là tout le problème. Morphy évoque la même question. Ces deux auteurs ont en fait une position très semblable: ils jugent que les artefacts artistiques ont des effets sur les personnes au voisinage desquelles ils se trouvent (l'art ne se contente pas de «dire 》, il «fait 》) - et en ce sens Gell a offert un changement de perspective intéressant -, mais ils n'y voient pas une raison suffisante pour rejeter l'approche sémiologique classique. Bien au contraire, si les artefacts artistiques ont un effet, c'est en raison de leur signification, de la manière dont ils sont «lus », selon Layton (2003: 460); alors que Morphy souligne que les objets étudiés par Gell sont ceux qui ont une «importance symbolique 》 (2009: 8) et conclut que «la composante sémantique de l'art peut être essentielle à son mode d'agir sur le monde » (2009: 14; voir aussi Campbell 2001 : 123). Par conséquent, au lieu d'éliminer de manière arrogante les acquis de l'approche sémiologique, de l'étude classique de l'art, il est suffisant de les utiliser avec une finalité légèrement différente; et là où Gell a tort, c'est dans son rejet initial, puis dans le peu d'atten-

\section{$\bullet$}

3. Voir par exemple Pinney et Thomas 2001 et Coquet, Derlon et JeudyBallini 2005. tion qu'il accorde aux conventions, à la signification, au contexte de production des œuvres, etc. C'est une interprétation de Gell peut-être justifiable, mais qui représente aussi la manière la plus conservatrice d'incorporer sa pensée aux perspectives existantes. L'auteur est alors domestiqué par les anthropologues de l'art alors qu'il espérait radicaliser le champ d'étude.

À l'inverse, d'autres anthropologues ont souligné que Gell avait peut-être ouvert la voie à une nouvelle perspective théorique, mais en lui reprochant d'être resté prisonnier de certains préjugés. En général, ils n'étaient, et ne sont pas, spécialistes de l'art et, précisément, ils utilisent l'approche de Gell pour parler des artefacts en général (réalisant, en quelque sorte, les craintes des auteurs discutés précédemment). Un bon exemple en est donné par Thinking Through Things, un volume collectif coordonné par Amiria Henare, Martin Holbraad et Sari Wastell (2007) : Gell a, selon eux, réalisé un grand pas en avant en développant une théorie qui traite les objets comme des personnes, en leur attribuant une agentivité. Mais il n'est justement pas allé assez loin parce que, dans son approche, les choses ne sont que des «agents secondaires » auxquels des humains délèguent et imputent agentivité et intentionnalité. Autrement dit, les choses sont «comme des personnes», sans en être complètement. II est intéressant de s'arrêter un instant sur certaines pages de L'Art et ses agents où cette question est abordée. D'une part, le point de vue de Gell est « exclusivement relationnel » (1998 : 22, 2009 [1998] : 28) : une entité est un agent, non pas en soi, mais dans la mesure où elle exerce une action sur un patient (qui n'est, aussi, un patient que de ce point de vue), et cela correspond à «des moments fugaces et parfois critiques de la vie sociale » (ibid.). C'est pour cette raison qu'il n'y a pas de mysticisme

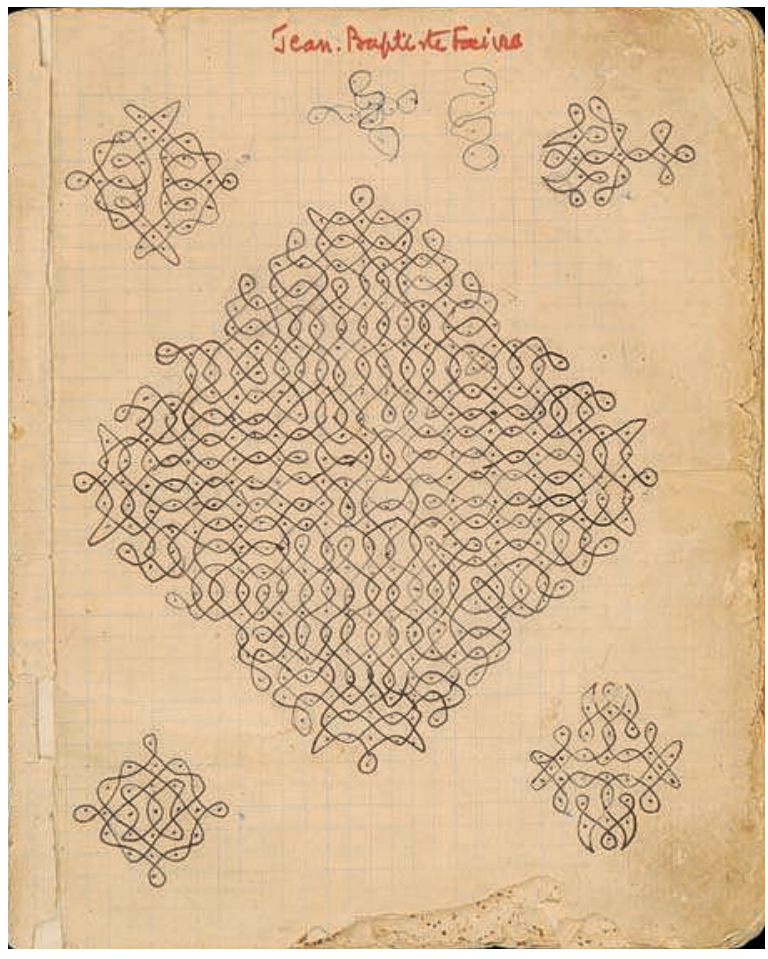

Fig. 1 Kolam, livre de dessin de sol, Pondichéry (c) musée du quai Branly. 
à considérer qu'une personne humaine peut être un patient par rapport à un artefact artistique (par exemple, on souffre des conséquences d'une caricature particulièrement méchante et sentie). Mais, d'autre part, l'agentivité semble souvent être un concept bien plus substantiel, que l'on peut alors définir comme «une capacité interne de l'esprit d'avoir des intentions et d'agir en conséquence ${ }^{4}$ » (Ingold et Hallam 2007: ?). D'un point de vue strict, seules les personnes humaines ont des intentions en tant qu'états mentaux. Même si Gell, en discutant des idoles, souligne que l'imputation d'intentionnalité ne dépend au fond pas de la nature biologique de l'entité en question et revient à une conception relationnelle (1998:123, 2009 [1998] : 151], il veut avant tout montrer que cette imputation n'est pas irrationnelle ou stupide. Les artefacts ne sont en réalité que des « agents secondaires » (1998: 20, 2009 [1998] : 26) dans la mesure où, en étant le produit d'une action intentionnelle, ils relaient cette intentionnalité agissante, celle de l'artiste (caricatures, motif labyrinthique; sur les pièges, voir aussi Gell 1999 [1996]: 201) ou du prototype (tableau de Louis XIV par Hyacinthe Rigaud), ou bien encore parce qu'on leur impute une intentionnalité (comme dans le cas des idoles]. Or il semble qu'aucune des deux facettes de la théorie de Gell ne satisfasse Henare, Holbraad et Wastell : ni la théorie relationnelle, parce qu'elle ne touche pas à la nature des choses; ni l'idée que les artefacts ne peuvent être que des agents secondaires, car elle maintient alors l'opposition entre sujet et objet. C'est précisément ce que ces auteurs rejettent, en voulant penser les choses « comme des significations sui generis » (2007 : 3), critiquant ainsi radicalement l'idée qu'il n'y a qu'un seul monde (où les personnes et les choses sont irréductibles] et différentes visions du monde qui conduisent certains à croire que le monde est différent de ce qu'il est réellement : il y a au contraire différents mondes (ibid. : 10 - c'est alors précisément l'opposé de Morphy).

Je ne veux pas rentrer dans le détail de cette approche ambitieuse et contestable, mais m'intéresser à seulement un aspect de la critique. Henare, Holbraad et Wastell reprochent à Gell, malgré certaines avancées, de supposer que l'«agentivité [des choses] trouve son origine dans l'esprit de leurs créateurs » (2007 : 17), ce qui reproduit l'opposition entre sujets et objets. James Leach atteint la même conclusion, soulignant que, chez Gell, «les nonhumains ne peuvent être des agents que par procuration » (2007 : 183), ce qui vient selon lui d'un biais typiquement euro-américain en faveur des esprits individuels (individual minds), sièges de la virtuosité technique et de la créativité. D'une manière relativement surprenante, on retrouve exactement la même critique chez Tim Ingold, qui, traitant de la créativité, refuse de l'identifier à l'agentivité (comme propriété interne d'esprits individuels] car il veut lui aussi dépasser « l'opposition moderne du sujet et de l'objet, ou du mental et du matériel » (2007 : 52), alors qu'on s'y enferme lorsqu'on envi-

\section{- $\bullet$}

4. Le terme agentivité, ou agency, est rendu particulièrement ambigu par son usage à la fois en philosophie morale et en science cognitive, avec des sens fort différents, comme l'a souligné Maurice Bloch lors de la présentation de L'Art et ses agents organisée au musée du quai Branly (12 juin 2009). En témoignent les multiples traductions du terme en français : agentivité, mais aussi agencéité ou agence, alors que certains conservent agency et d'autres choisissent de parler plus simplement d'intentionnalité (dont Bloch 1999).

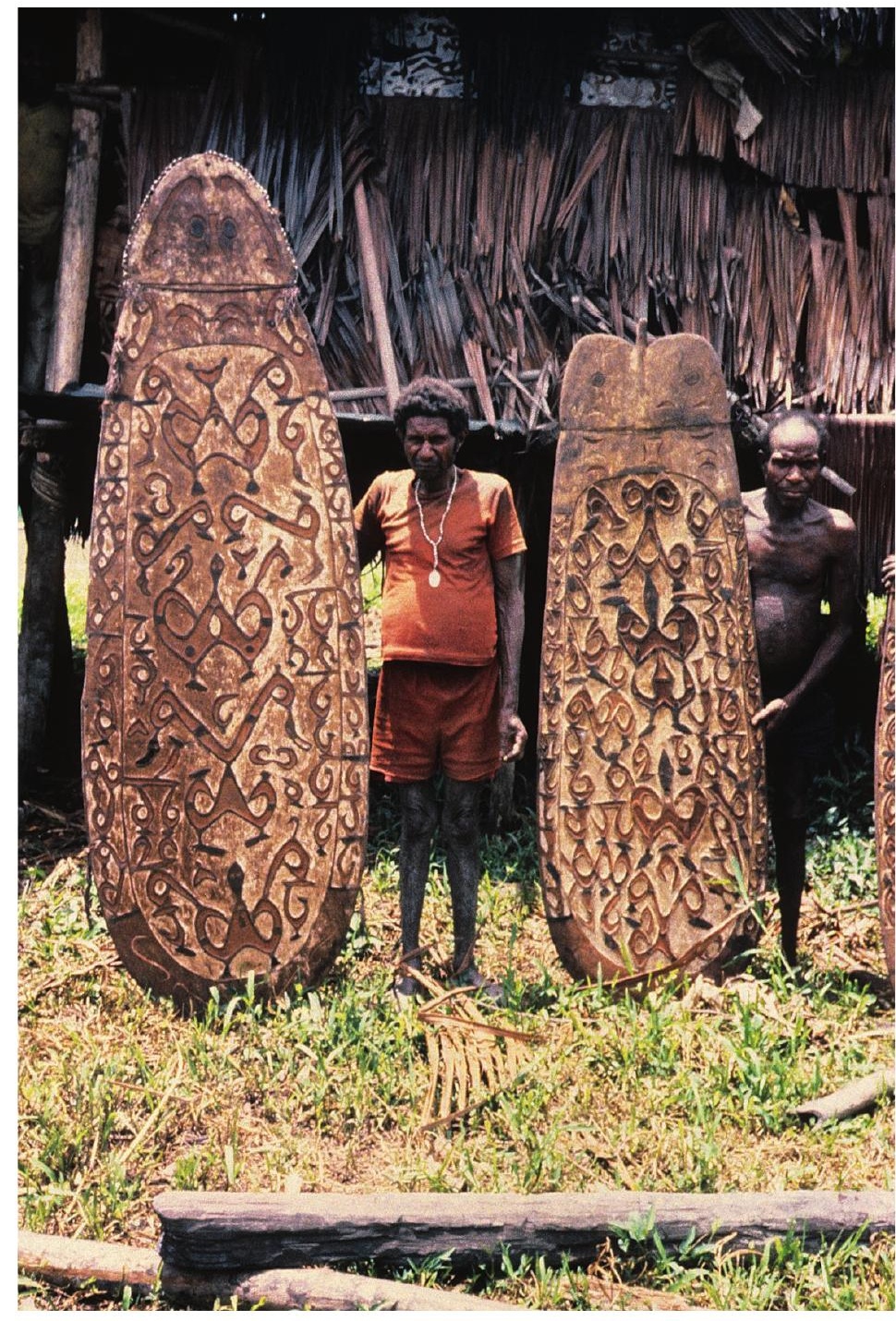

Fig. 2 Hommes asmat et boucliers sculptés

(c) musée du quai Branly, photo Todd Barlin.

sage l'agentivité comme un attribut des entités du monde (même en $y$ incluant des choses et non plus seulement des personnes]. Au contraire, selon lui, l'agentivité «possède» les entités, c'est «le flux génératif du monde lui-même dans sa concrescence continuelle » (ibid.). De manière plus précise et ethnographique, il s'appuie sur la reprise par un des contributeurs de Creativity and cultural improvisation du cas des kolam discuté par Gell (cf. fig. 1). Ce dernier interprète ces motifs apotropaïques indiens (« des papiers tue-mouche où viennent s'engluer les démons »; 1998: 84, 2009 [1998] : 104] comme des «pièges à pensée » (mind-traps; voir aussi Gell 1999 [1996]) dans la mesure où notre esprit serait incapable de saisir la logique du motif, tout en étant conscient qu'il y en a une - ce qui fournit à Gell son interprétation des motifs abstraits et de l'art décoratif en général. Amar S. Mall (2007) offre effectivement une perspective intéressante sur l'interprétation de ces motifs. Un des deux types de kolam (où on entoure les points disposés sur le sol au lieu de les rejoindre] n'est en ef- 


\section{CHRONIQUE SCIENTIFIQUE NOTES CRITIOUES}

fet pas le strict produit d'une conception préalable : d'une part, on dit que le motif «advient » lorsqu'il est produit; d'autre part, les femmes reconnaissent qu'elles peuvent modifier au cours du tracé le motif qu'elles avaient prévu de réaliser. Ce phénomène «situe la "créativité" quelque part entre les intentions de l'exécutant et cette capacité "auto-génératrice" de la forme kolam » (ibid. : 70). Ingold reprend alors cette analyse pour critiquer Gell et conclure que «ce serait une grande erreur que de regarder toute sorte de motif comme la réalisation d'un assemblage cognitif préconçu, ou comme l'incarnation d'une "conception" qui est située dans l'esprit du fabriquant», tout comme de le voir comme un puzzle intellectuel pour ceux qui le regardent (ibid. : 49).

\section{Agentivité et cognition}

Malgré la diversité des critiques, tous ces auteurs se rejoignent pour relever le primat analytique accordé aux esprits (minds) de personnes individuelles. Nombre des développements de Gell ne se réduisent certes pas à cette question, et c'est principalement pour cette raison qu'il a été critiqué, mais je souhaite précisément rendre explicite l'importance - à mon avis centrale - de la cognition dans L'Art et ses agents. C'est un élément qui pose problème à Ingold et Hallam (2007), ainsi qu'à Henare et alii (2007), alors qu'il n'y a, a priori, pas de conflit avec une anthropologie de l'art plus classique, bien que subsiste une différence importante. Pour Layton ou Morphy, par exemple, les créateurs ou fabricants sont avant tout des représentants d'une culture; ils suivent des conventions, des croyances, des symboles, etc., qui ne sont pas universels, et en fonction desquels les artefacts sont «lus » ou bien ont un effet. Si Gell n'écarte pas entièrement cette question, elle semble chez lui secondaire par rapport à celle des effets cognitifs - en termes relativement universels - des artefacts sur les destinataires. La question du rapport de Gell au cognitivisme ou à la cognition est assez complexe et je ne vais en aborder qu'un aspect $^{5}$. En commentant Gell, Maurice Bloch (1999, 2009

\section{- $\bullet$}

5. Je ne vais notamment pas discuter son chapitre final, qui cherche à montrer la manière dont une œuvre, individuelle ou collective, matérialise des processus cognitifs. Morten Axel Pedersen (2007) le suit dans cette voie, en étayant son argumentation à l'aide de travaux récents des sciences cognitives sur le sujet.

6. II s'agit de la recension de l'édition anglaise (1999), reproduite en préface de l'édition française (2009).
[19996]] évoque essentiellement les théories cognitives qui concernent la faculté permettant aux humains «d'imaginer ce qui se passe dans l'esprit des autres » (theory of mind), ce qu'il mobilise notamment pour rendre compte de l'idée d'abduction? d'agentivité et pour l'analyse des idoles qui possèdent ou possèderaient une intentionnalité (dans le premier cas, les «autres» sont des humains, dans le second il s'agit d'artefacts ou plutôt de divinités]. Toutefois, il ne me semble pas relier cette question à celle des caractéristiques matérielles et formelles des artefacts, et demande même: les objets «n'expriment-ils pas l'intentionnalité d'une manière toute différente de celle des êtres vivants? 》 (1999: 127, 2009 [1999]: XV). Or il s'agit précisément, à mon avis, d'un aspect central de l'ouvrage. Gell développe brièvement les théories élaborées par les philosophes et les cogniticiens pour expliquer comment les humains appréhendent le comportement d'autrui, et il distingue théories externalistes (autrui suit une conduite de vie, des règles] et internalistes [autrui possède des états internes qui génèrent son comportement). Mais s'il souligne que les premières peuvent être pertinentes pour son sujet (par exemple, l'idole ne réagit pas lorsqu'une souris lui court dessus parce qu'elle a atteint un état d'autocontrôle quasi divin), il privilégie les secondes, qu'il considère plus fé-

condes, dans la mesure où on peut les mettre en relation avec la structure matérielle des idoles en tant qu'artefacts. Dans la plupart des cas, les idoles sont constituées de manière à suggérer un «intérieur», à établir implicitement un rapport contenu/contenant qui pointe vers des «états internes», vers une intentionnalité (Gell 1998 : 132, 2009 [1998] : 163] : il s'agit de pierres conservées dans un temple obscur et fermé, du to'o polynésien enveloppé de toile d'écorce - et parfois de phénomènes plus complexes, comme l'idole de Jagannath à Puri, qui est une statuette en bois, peinte et enveloppée de tissus, contenant une substance vitale secrète transmise de

\section{- $\bullet$}

?. L'abduction est un procédé logiquement faux, mais pratiquement nécessaire, par lequel on remonte d'un effet (l'indice, l'œuvre d'art, par exemple) à sa cause (l'intentionnalité agissante d'un artiste, d'un commanditaire, d'une divinité, etc.). L'exemple typique consiste à déduire l'existence d'un feu de la présence de fumée, ce qui est sans doute statistiquement juste... 
statue en statue (probablement une pierre)

Ce travail me semble exemplaire de la manière dont on peut mobiliser dans l'analyse des contraintes cognitives ou des processus psychologiques universels sans pour autant sacrifier la richesse ethnographique propre à des artefacts et à des contextes particuliers. L'analyse que fait Gell de l'art décoratif comme une «technique d'enchantement » (technology of enchantment ; 1999 [1992]] en est un autre exemple, de même que son traitement du style ${ }^{8}$ en termes de traits «psychologiquement saillants » (1998: 157, 2009 [1998] : 191). Parlant de cette dernière question, il souligne qu'« une description de style "culturelle" serait un compte rendu abstrait des propriétés des œuvres d'art à la lumière de leur capacité à thématiser et à rendre saillants (d'un point de vue cognitif) des paramètres culturels essentiels » (1998: 159, 2009 [1998] : 194). Cette saillance psychologique est enracinée, selon Gell, dans la relation qu'un artefact entretient avec l'ensemble des autres artefacts relevant du même style, c'est-à-dire qu'il existerait un effet cognitif lié au fait d'être une œuvre parmi d'autres, membre d'un ensemble stylistique (qu'il s'agisse du style d'un artiste ou d'une culture) plutôt qu'artefact isolé. Dans les îles Marquises, il s'agit du fait que tous les motifs sont des transformations les uns des autres, plus précisément sont le produit des transformations (formelles] minimales permettant de les différencier.

Gell utilise à de nombreuses reprises un vocabulaire renvoyant à la cognition, plus ou moins explicitement, et fait parfois référence à des travaux sur ce thème (théories internalistes ou externalistes par exemple). Toutefois, il n'a jamais été un cognitiviste, et la manière dont il utilise ces concepts a pu mettre certains de ses lecteurs mal à l'aise. Bloch (1999: 126-127, 2009 [1999] : XIV-XVI] souligne qu'il laisse certains points dans l'ombre, et Layton (2003) ainsi que Morphy (2009) sont beaucoup plus agressifs, notamment lorsque Gell utilise sa propre expérience pour justifier les effets psychologiques d'une œuvre d'art : «Gell semble supposer que sa propre interprétation subjective d'un motif est à la fois universelle et culturellement pertinente, mais il ne fournit aucune preuve » (Morphy 2009 : 13) ; «je trouve que cette étape de l'argumentation de Gell est affaiblie par son usage de la stratégie victorienne qui consiste à s'imaginer dans la position d'un membre d'une autre culture » (Layton 2003 : 457). II est vrai que, sur un certain nombre de points, Gell a eu des intuitions qui paraissent justes ou au moins intéressantes, mais à l'appui desquelles il ne fournit pas de données scientifiques. Ce thème aurait précisément pu être poursuivi par d'autres chercheurs ${ }^{9}$. Cependant, la cognition ou les effets psychologiques des artefacts artistiques sont réellement au cœur de l'argumentation de L'Art et ses agents - même si Gell ne semble parfois les évoquer qu'en passant, en amateur. Des anthropologues de l'art comme Layton ou Morphy soulignent que c'est uniquement à travers leur signification symbolique, ou en tant que système de

\section{-}

8. Gell traite plus globalement du rapport entre style et culture, à partir d'une étude des îles Marquises.

9. Bien que la perspective soit différente et que le débat avec Gell ne soit pas vraiment engagé, on peut trouver des échos de ces préoccupations dans les ouvrages récents de Barbara Maria Stafford (2007) et Carlo Severi (200?). signes, que les artefacts artistiques exercent une agentivité - d'où leur désir de montrer qu'on ne peut se passer d'une analyse sémiologique. Je pense, au contraire, qu'ils ont dans l'argumentation de Gell un effet essentiellement cognitif. II faut toutefois souligner à ce sujet que Gell radicalise souvent sa position théorique dans ses déclarations préliminaires afin de souligner l'originalité de son approche, mais qu'il n'épure pas pour autant son analyse de ce qu'on appelle traditionnellement le contexte ethnographique (conventions, conceptions, symboles, croyances, etc.) : il se contente de subordonner ces aspects aux effets proprement cognitifs des artefacts artistiques. C'est par exemple frappant dans son analyse du style des îles Marquises, qui est intrinsèquement lié au fait qu'il s'agit de formes artistiques de «magnification de la personne » (1998: 199, 2009 [1998] : 239, traduction modifiée ; c'est un thème qu'il avait déjà traité auparavant, voir Gell 1993; cf. fig. 3). C'est ainsi que Gell explique la manière dont les protections magiques sont multipliées par ajout de «dos » à des œuvres en deux dimensions, en dédoublant des motifs (et en les transformant adéquatement) pour qu'ils protègent la personne de toutes parts. On retrouve un phénomène similaire pour les marchepieds d'échasse utilisés lors de duels rituels, ainsi que les manches d'éventail, dont les motifs renvoient à la multiplication numérique des soutiens politiques des chefs pourvus de tels ustensiles. Ce type d'analyse en termes de contexte culturel n'est clairement pas inutile, mais ne constitue pas une fin en soi : il ne représente qu'une étape et ne préjuge rien de la manière dont les motifs se transforment en se multipliant - or c'est ce qui intéresse Gell dans l'analyse du style.

\section{L'art en question}

La plupart des anthropologues de l'art ici évoqués ont voulu montrer que l'analyse sémiologique était nécessaire parce qu'elle permet de comprendre les effets propres des œuvres d'art et en même temps restaure la spécificité de ces dernières. À l'inverse, les autres anthropologues qui discutent Gell ont parfois cherché à étendre et dépasser son approche, en critiquant le privilège accordé aux esprits individuels, mais aussi en parlant des «choses » en général (Henare et alii 2007) ou de la «matérialité», ce qui les a conduits à dissoudre entièrement l'art comme objet ou catégorie pertinente. À ce sujet, il est remarquable que la plupart des auteurs ayant contribué au volume Materiality (Miller 2005] associent systématiquement l'approche de Gell et celle de Bruno Latour, comme deux tentatives de déstabiliser l'opposition entre des sujets (humains) et des objets (naturels ou matériels), qui sont en général radicalement séparés et dans une relation asymétrique. Or Latour traite essentiellement de la science, d'entités hybrides produites dans les laboratoires - rien à voir avec l'art (voir Latour 1991). Mais si leurs perspectives me semblent relever d'interrogations ou d'insatisfactions parentes, elles sont radicalement différentes, précisément en raison du rôle de la cognition chez Gell. Des artefacts quelconques étudiés dans la perspective de Gell peuvent être vus comme une extension des personnes qui les ont produits, en réalisant une «abduction d'agentivité »; on peut ne pas les considérer comme des choses, mais plutôt «comme des personnes » ayant des effets. Mais ce type d'analyse est antérieur à l'ouvrage de Gell, et ne me semble 


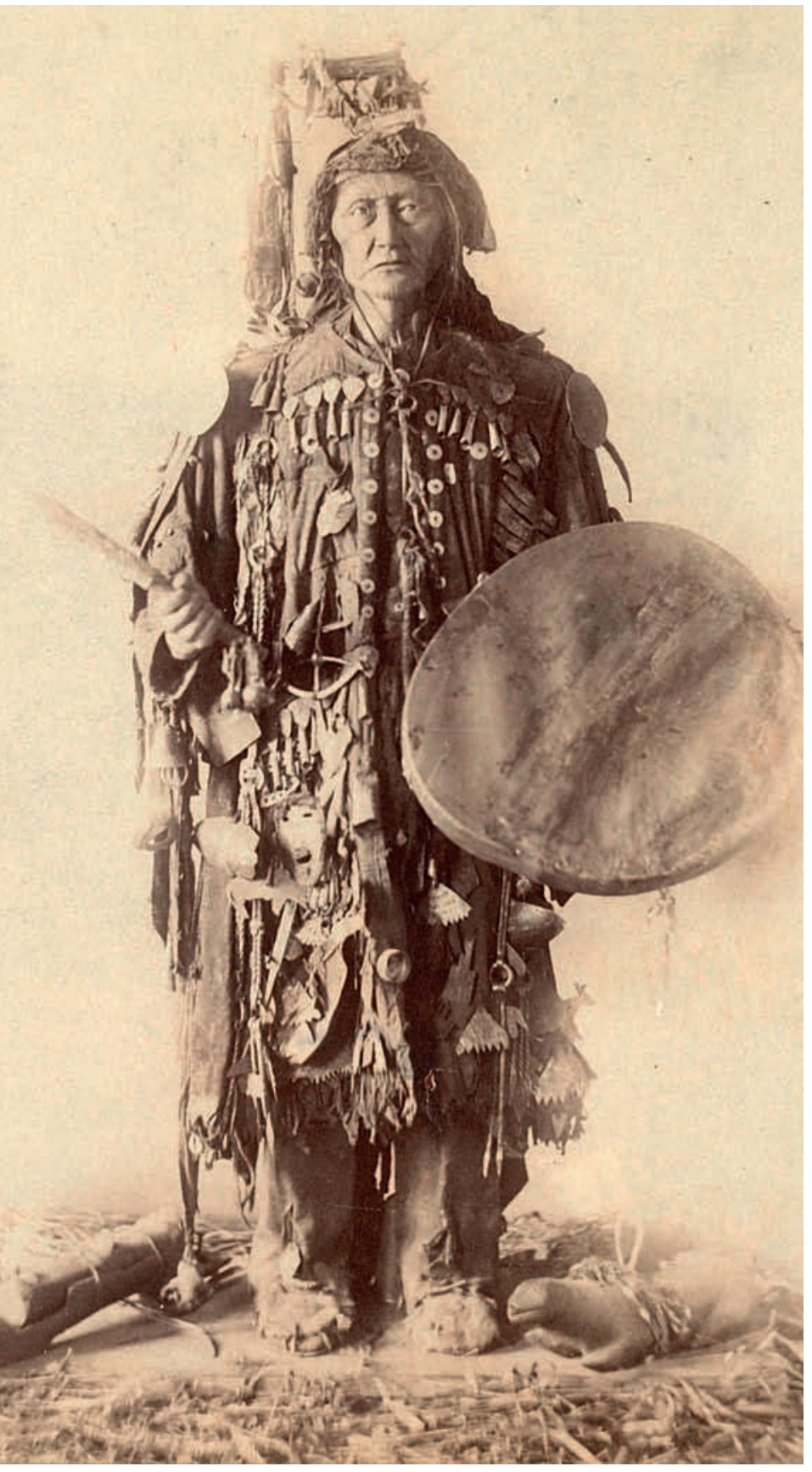

Fig. 4 Une chamane toungouze, Transbaïkalie, Russie (1850-1899) (c) musée du quai Branly, photo Kobizeff-Angarski.

pas avoir la même portée : plus de dix ans avant, Arjun Appadurai écrivait déjà que, dans de nombreuses sociétés, les «choses》 ont conservé la «faculté des personnes à agir» (1986: 4). Au contraire, chez Gell, tous ces phénomènes sont intimement liés aux caractéristiques matérielles et formelles des artefacts qu'il étudie, parce que ces derniers ont des effets cognitifs sur leurs destinataires. On peut adopter une telle perspective lorsqu'on étudie, par exemple, des costumes chamaniques hétéroclites qui renvoient à la nature polymorphe et transformationnelle des esprits (tout en la faisant advenir lors des rituels), et qui sont recouverts de nœuds correspondant à chaque cure particulière, rendant alors visible l'ensemble des interactions thérapeutiques du chamane qui officie (Pedersen 2007; cf. fig. 4). Mais cela a en revanche beaucoup moins de sens lorsqu'on se contente de dire que les vêtements occidentaux appropriés par les Amérindiens exercent une agentivité liée à leur altérité originelle. Et ce n'est pas un hasard si les premiers sont généralement considérés comme des exemplaires d'art ethnographique dignes d'être exposés dans les musées, et non les seconds. Gell prend grand soin, au début de L'Art et ses agents, de ne pas définir l'«art 》 de manière essentialiste, soulignant qu'il propose une définition théorique relationnelle - mais il ajoute qu'il discutera essentiellement des objets habituellement identifiés comme artistiques [1998 : ?, 2009 [1998] : 8). Je ne tiens pas, non plus, à proposer une définition de l'art, de quelque manière que ce soit. Mais je voudrais souligner que, malgré cette absence de définition, l'approche de Gell «marche »-au sens où elle est féconde, intéressante, originale - particulièrement lorsque les artefacts étudiés sont cognitivement complexes, c'est-à-dire lorsqu'ils ne sont pas aisément appréhensibles par l'esprit humain, lorsqu'ils possèdent des traits psychologiquement saillants, lorsque l'abduction d'agentivité ne va pas de soi $^{10}$. Et, de manière générale, c'est une caractéristique que possèdent les artefacts artistiques. Ceci me semble bien évidemment vrai d'un tatouage marquisien, mais également d'artefacts qui peuvent être plastiquement ou techniquement très simples mais cognitivement complexes (par exemple un disque noir qui n'est pas positionné au centre d'un carré blanc, ou bien une pierre ronde sommairement percée d'un côté). C'est aussi ce qui fait que, si un artefact (idole) et un être humain (déesse vivante ou médium) peuvent être traités de la même manière par l'anthropologie conçue par Gell (1998: 153, 2009 [1998] : 186], ce n'est précisément pas n'importe quel être humain qu'il est pertinent de voir ainsi, mais par exemple une petite fille décorée, et sur le front de laquelle on a peint un troisième œil.

Olivier Allard

Cambridge University oli.allard@cantab.net

\section{- $\bullet$}

10. Dans une publication précédente, alors que son approche n'était encore qu'ébauchée, Gell définissait comme œuvre d'art potentielle tout objet qui assouvit l'examen intense des destinataires parce qu'il «incorpore des intentionnalités qui sont complexes, exigent une attention soutenue, et sont peut-être difficiles à reconstruire entièrement » (1999 [1996] : 211). Dans une telle formulation, le caractère novateur de cette approche provient davantage de l'idée de complexité et de difficulté que de l'hypothèse selon laquelle des intentionnalités sont «incorporées 》 dans les artefacts. 


\section{Bibliographie}

\section{Appadurai, Arjun}

1986 « Introduction: commodities and the politics of value », in Arjun Appadurai (éd.), The Social Life of things. Commodities in cultural perspective. Cambridge, Cambridge University Press.

\section{Bıосн, Maurice}

1999 « Une nouvelle théorie de l'art. À propos d'Art and Agency d'Alfred Gell », Terrain 32 : 119-128.

2009 [1999] «Une nouvelle théorie de l'art 》, in Alfred Gell, L'Art et ses agents. Une théorie anthropologique. Bruxelles, Les presses du réel [«Fabula 》] : VII-XVII.

\section{Boyer, Pascal}

1988 Barricades mystérieuses et pièges à pensée. Introduction à l'analyse des épopées fang. Nanterre, Société d'ethnologie.

\section{CAmpbeli, Shirley}

2001 « The captivating agency of art: many ways of seeing », in Christopher Pinney et Nicholas Thomas (éd.), Beyond aesthetics. Art and the technologies of enchantment. Oxford, Berg : 117-135.

Coonet, Michèle, Derion, Brigitte et Jeudy-Ballini, Monique (éd.)

2005 Les Cultures à l'œuvre. Rencontres en art. Paris, Biro-éditions de la Maison des sciences de l'homme.

\section{GeLL, Alfred}

1993 Wrapping in Images. Tattooing in Polynesia. Oxford, Clarendon Press. 1998 Art and Agency. An Anthropological Theory. Oxford, Clarendon Press. 1999 [1992] «The Technology of Enchantment and the Enchantment of Technology », in Alfred Gell, The Art of Anthropology. Essays and Diagrams. Londres, The Athlone Press : 159-186.

1999 [1996] « Vogel's Net. Traps as artworks and artworks as traps », in Alfred Gell, The Art of Anthropology. Essays and Diagrams. Londres, The Athlone Press : 187-214.
2009 [1998] L'Art et ses agents. Une théorie anthropologique. Bruxelles, Les presses du réel [«Fabula 》].

Henare, Amiria, Holbraad, Martin et WASTELL, Sari

2007 «Introduction », in Amiria Henare, Martin Holbraad et Sari Wastell (éd.], Thinking through things. Theorising artefacts ethnographically. Londres, Routledge : 1-31.

\section{IngOLD, Tim}

2007 «Introduction (part one) », in Tim Ingold et Elizabeth Hallam (éd.), Creativity and cultural improvisation. Oxford, Berg : 45-53.

\section{Ingold, Tim et Hallam, Elizabeth} 2007 « Creativity and Cultural Improvisation: an Introduction », in Tim Ingold et Elizabeth Hallam (éd.), Creativity and cultural improvisation. Oxford, Berg : 1-24.

\section{Latour, Bruno}

1991 Nous n'avons jamais été modernes. Essai d'anthropologie symétrique. Paris, La Découverte.

LAYTON, Robert

2003 «Art and Agency: a reassessment 》, Journal of the Royal Anthropological Institute $9(3): 447-464$.

LEACH, James

2007 « Differenciation and encompassment. A critique of Alfred Gell's theory of creativity », in Amiria Henare, Martin Holbraad et Sari Wastell [éd.], Thinking through things. Theorising artefacts ethnographically. Londres, Routledge : 167-188.

\section{Lévi-Strauss, Claude}

1950 «Introduction à l'œuvre de Marcel Mauss », in Marcel Mauss, Sociologie et anthropologie. Paris, PUF : IX-LII.

\section{MaLL, Amar S.}

2007 « Structure, Innovation and Agency in Pattern Construction : The Kolam of Southern India », in Tim Ingold et Elizabeth Hallam (éd.), Creativity and cultural improvisation. Oxford, Berg : 55-78.
Mauss, Marcel

1950 [1923-1924] «Essai sur le don », Sociologie et anthropologie. Paris, PUF : 143-279.

\section{Miller, Daniel}

2000 «Truly intellectual traps », Times Higher Education, 12 mai.

Miller, Daniel (éd.)

2005 Materiality. Durham, Duke University Press.

\section{MorpHy, Howard}

2009 «Art as a mode of action. Some problems with Gell's Art and Agency », Journal of material culture 14[1] : 5-2?

\section{Pedersen, Morten Axel}

2007 « Talismans of thought. Shamanist ontologies and extended cognition in Northern Mongolia », in Amiria Henare, Martin Holbraad et Sari Wastell (éd.), Thinking through things. Theorising artefacts ethnographically. Londres, Routledge : 141-166.

Pinney, Christopher et Thomas, Nicholas (éd.)

2001 Beyond aesthetics. Art and the technologies of enchantment. Oxford, Berg.

\section{Severi, Carlo}

2007 Le Principe de la chimère. Une anthropologie de la mémoire. Paris, éditions Rue d'Ulm-musée du quai Branly.

\section{Sмith, Pierre}

1979 «Aspects de l'organisation des rites », in Michel Izard et Pierre Smith (éd.), La Fonction symbolique. Paris, Gallimard : 139-170.

\section{StAFFord, Barbara Maria}

2007 Echo Objects. The Cognitive Work of Images. Chicago, The University of Chicago Press.

Strathern, Marilyn

1988 The Gender of the gift. Problems with women and problems with society in Melanesia. Berkeley, University of California Press. 